\title{
Estudo comparativo entre transmissões automotivas automáticas e manuais
}

\section{Comparative study between automatic and manual automotive transmissions}

DOI: $10.46814 /$ lajdv3n5-003

Recebimento dos originais: 01/07/2021

Aceitação para publicação: 31/08/2021

\section{Caik Gabriel Cavalcante Soares}

Engenheiro Mecânico

Departamento de Engenharia Mecânica, Universidade do Estado do Amazonas, Manaus, AM Ave. Darcy Vargas 1200, Parque 10, Manaus AM, CEP 69050-020

E-mail: cgcs.eng16@uea.edu.br

\section{Gilberto Garcia del Pino \\ Doutor}

Departamento de Engenharia Mecânica, Universidade do Estado do Amazonas, Manaus, AM

Ave. Darcy Vargas 1200, Parque 10, Manaus AM, CEP 69050-020

E-mail: gpino@uea.edu.br

\author{
Abderrezak Bezazi, \\ Doutor
}

Laboratoire de Mécanique Appliquée des Nouveaux Matériaux (LMANM), Université 8 Mai 1945 BP 401, Guelma 24000, Algér

E-mail: ar_bezazi@yahoo.com

\section{Arlindo Pires Lopes}

Doutor

Departamento de Engenharia Mecânica, Universidade do Estado do Amazonas, Manaus, AM

Ave. Darcy Vargas 1200, Parque 10, Manaus AM, CEP 69050-020

E-mail: arlindopireslopes@gmail.com

\section{Aristides Rivera Torres}

Doutor

Departamento de Engenharia Mecânica, Universidade do Estado do Amazonas, Manaus, AM

Ave. Darcy Vargas 1200, Parque 10, Manaus AM, CEP 69050-020

E-mail: artorres@uea.edu.br

\section{Antônio Claudio Kieling}

Doutor

Departamento de Engenharia Mecânica, Universidade do Estado do Amazonas, Manaus, AM

Ave. Darcy Vargas 1200, Parque 10, Manaus AM, CEP 69050-020

E-mail: akieling@uea.edu.br

\section{Marcos Dantas do Santos}

Mestre

Departamento de Engenharia Mecânica, Universidade do Estado do Amazonas, Manaus, AM

Ave. Darcy Vargas 1200, Parque 10, Manaus AM, CEP 69050-020

E-mail: mdsantos@uea.edu.br 


\title{
Gabriel Federico Rivero Llerena
}

\author{
Doutor
}

Departamento de Engenharia Mecânica, Universidade do Estado do Amazonas, Manaus, AM Ave. Darcy Vargas 1200, Parque 10, Manaus AM, CEP 69050-020

E-mail: gllerena@uea.edu.br

\section{RESUMO}

O mercado automobilístico vem intensificando a criação e a aplicação de novas tecnologias veiculares. Nesse sentindo, entre os elementos que sofreram notável evolução, destacam-se as transmissões automotivas, que são objetos fundamentais na dinâmica veicular, pois contribuem para o deslocamento do automóvel e, além disso, são ofertadas no mercado nas mais diversas variações. O objetivo deste trabalho foi elaborar uma análise técnica comparativa entre os principais modelos de transmissões presentes no mercado: automática e manual. Primeiramente, foi realizada uma comparação em relação à parte mecânica estudando os principais cálculos associados ao desempenho de modelos equipados com esses mecanismos e foi realizada uma análise econômica. Os resultados mostram um relacionamento de aspectos que englobam desempenho, manutenção e consumo entre esses componentes com bons resultados.

Palavras-chave: Transmissão Veicular, Indústria Automobilística, Desempenho Automotivo.

\begin{abstract}
The automobile market has been intensifying the creation and application of new vehicle technologies. In this sense, among the elements that have undergone notable evolution, automotive transmissions stand out, which are fundamental objects in vehicle dynamics, as they contribute to the displacement of the automobile and, in addition, are offered in the market in the most diverse variations. The objective of this work was to elaborate a comparative technical analysis between the main transmission models present in the market: automatic and manual. First, a comparison in relation to the mechanical part was carried out, studying the main calculations associated with the performance of models equipped with these mechanisms, and an economic analysis was carried out. The results show a relationship of aspects that encompass performance, maintenance and consumption between these components with good results.
\end{abstract}

Keywords: Vehicle Transmission, Automotive Industry, Automotive Performance

\section{INTRODUÇÃO}

A transmissão é um dos componentes veiculares com maior evolução e complexidade desde o início da indústria automobilística, que ocorreu há mais de um século, pois é a responsável por transformar o torque produzido pelo motor em movimento para as rodas motrizes do automóvel, sendo, portanto, fundamental na dinâmica veicular, [1].

Segundo (Lechner; Naunheimer, (1999) [2], as primeiras ideias referentes a transmissão existem há mais de um milênio. A revolução industrial é marcada por um avanço em relação as técnicas de aprimoramento de multiplicação de força. As maquinas a vapor substituíram as forças animais e humanas. Segundo Padilha (2018) [3] o desenvolvimento de caixas de mudança de marchas estava 
diretamente ligado ao aprimoramento de máquinas a vapor, uma vez que as transmissões das forças geradas por esses equipamentos a vapor não eram suficientes.

As primeiras aplicações de transmissões com engrenagens começam na década de 1920 do século 19 (Lechner; Naunheimer, (1999) [2] e posteriormente o surgiu o diferencial, que é um elemento capaz de distribuir o torque entre semieixos em velocidades diferentes o que confere a possiblidade da realização segura de curvas. Saeed, 2018) [4]. Ao mesmo tempo foram criados novos tipos de ligas metálicas e processos fornecendo um incremento nas propriedades mecânicas dos materiais dos componentes que formam parte das transmissões possibilitando maiores velocidades de rotação e vida útil [5- 7].

Com o passar dos anos, uma variedade de transmissões tem sido desenvolvida e aplicada no mercado; existem modelos de câmbios que trabalham com conceitos estritamente mecânicos até elegantes peças eletroeletrônicas, que não exigem mais do condutor a operação com alavancas. Cada um desses tipos gerará diferentes desempenhos em relação ao consumo de combustível, tempo de resposta das rodas e vida útil. (SENAI, 2002). [8] Atualmente existem múltiplas ofertas de veículos no mercado, e, além disso, os modelos com câmbios automáticos caminham para uma posição majoritária em alguns anos. (Goiés, 2020) [09]. Há, portanto, cada vez mais necessidade de elucidação e esclarecimento acerca destes mecanismos ao condutor, principalmente, antes do momento da escolha do câmbio que irá equipar seu veículo.

A transmissão é um componente que fundamentalmente se vale do torque produzido pelo motor para gerar a rotação dos eixos do automóvel. Atualmente existem 4 principais tipos de transmissões: Manual, Automática, Automática Continuously Variable Transmission (CVT) e automatizada. Cada uma dessas opções possui uma dinâmica diferente, porém atuam com o mesmo objetivo. [09]

A transmissão manual é a mais popular no Brasil. Estima-se que, há 10 anos, 90\% dos carros novos produzidos no Brasil eram de câmbio manual. Além disso, por se tratar de uma mecânica mais simples, os veículos portadores de câmbios manuais são mais acessíveis financeiramente. O câmbio automático, portanto, surge como uma alternativa para uma direção mais confortável, porém mais custosa e passou a englobar veículos de categorias mais elevadas. Entretanto, na atual conjuntura, parece existir uma nova tendência no mercado: onde houve um aumento de $40 \%$ pela procura de carros automáticos de pequeno porte (conhecidos como carros "populares") e cerca de $95 \%$ dos proprietários de veículos automáticos não voltariam para o câmbio manual, indicando uma nova preferência do consumidor brasileiro.[09]

Cada tipo de transmissão influenciará em aspectos essenciais no desempenho do veículo como: consumo de combustível, durabilidade, periodicidade de manutenções, tempo de respostas das rodas entre outros (Jatco, 2018) [10]. Portanto, surge uma necessidade de elucidação quanto ao desempenho 
de cada modelo de transmissão e também um esclarecimento da proposta deste mecanismo para o condutor, uma vez que as diversas opções de câmbio podem gerar incertezas no momento da escolha de um veículo. A partir desse cenário, foi realizado um estudo dos princípios de funcionamento mecânicos dos câmbios automáticos e manuais realizando uma análise comparativa, fundamentada em suas vantagens e desvantagens, analisando aspectos: econômicos, como custo de manutenção e sua rotatividade, e mecânicos, como desempenho e tecnologia, considerando também que são importantes os aspectos relacionados com a sustentabilidade e a proteção do meio ambiente. [11- 14]

É importante destacar que as caixas de velocidades automotivas funcionam através de marchas, que são ordenadas por relações de transmissão. As caixas automotivas trabalham inicialmente com a multiplicação do torque e, após um certo momento, com a multiplicação da velocidade. Existem modelos de caixas que se valem de engrenagens. Nesse caso, a relação de transmissão será obtida pela razão entre os diâmetros de cada engrenagem ou, até mesmo, com as razões entre o número de dentes dos pares motores e movidos (Melconian, 2018) [15]. Já os veículos que não utilizam engrenagens, como os automóveis equipados com as transmissões continuamente variáveis, possuem relações de transmissão equivalente a razão do diâmetro de suas polias.

A potência é uma grandeza física associada a quantidade de trabalho realizado por tempo, onde trabalho é a energia transferida a um objeto ou através de um objeto. (Halliday, 2012) [16]. Outra forma de calcular a potência é através do produto entre torque pela velocidade angular. O rendimento consiste na razão existente entre a potência produzida por determinado objeto e a potência transferida. É fundamental para o estudo de sistemas não-conservativos, que são sistemas onde existem perdas de energia. Ou seja, o rendimento objetiva medir o aproveitamento dentro de um sistema, trata-se de uma grandeza adimensional e seu valor costumeiramente é dado em porcentagem.

Existem duas principais configurações de transmissão. Cada uma diz respeito a quem será o eixo motriz e onde estará localizado o motor, que é o responsável por determinar a "tração" veicular. As principais estruturas são: “com motor e transmissão longitudinal em linha posicionado a frente do veículo tracionando as rodas traseiras (Figura 1a) ou com motor transversal a frente do veículo tracionando as rodas dianteiras (Figura 1b). Existem ainda configurações com o motor traseiro, mas são menos comuns atualmente. [3]. 
Figura 1: Tipos de transmissões (Padilha, 2018) [3]. a)- com motor e transmissão longitudinal em linha posicionado a frente do veículo, b)- com motor transversal a frente do veículo

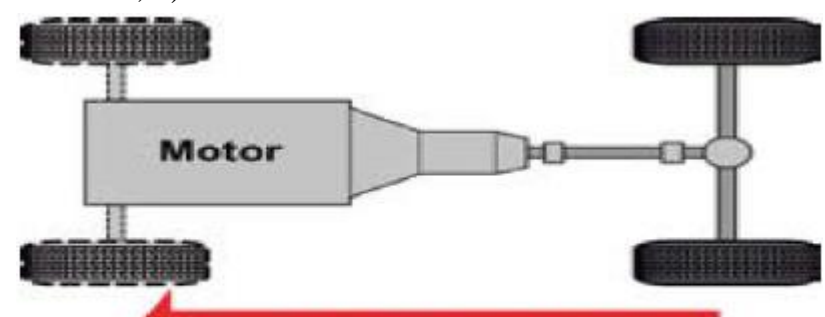

Sentido do movimento

a)

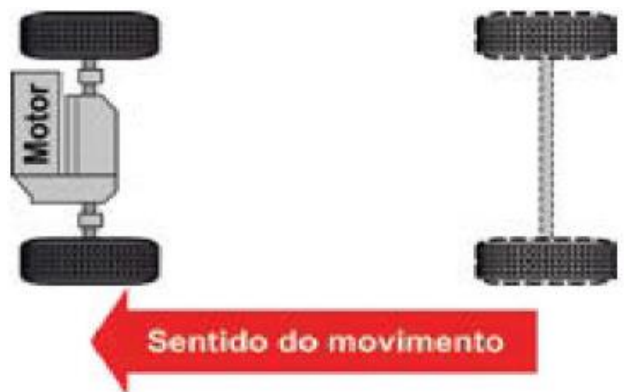

b)

Na figura 2, é mostrado um esquema dos principais componentes de um sistema de transmissão onde pode-se observar um mecanismo disposto em uma configuração longitudinal, ou seja, trata-se de um modelo de tração traseira. Os componentes principais são a embreagem que é exclusiva de veículos que possuem câmbios manuais, a caixa de velocidades onde existem as relações de engrenagens responsáveis pela multiplicação do torque, o eixo articulado presente exclusivamente em veículos com tração traseira, assim como o diferencial e os semieixos que são ligados finalmente as rodas motrizes do veículo. [17]

Figura 2: Sistema de transmissão. Adaptado de [17]

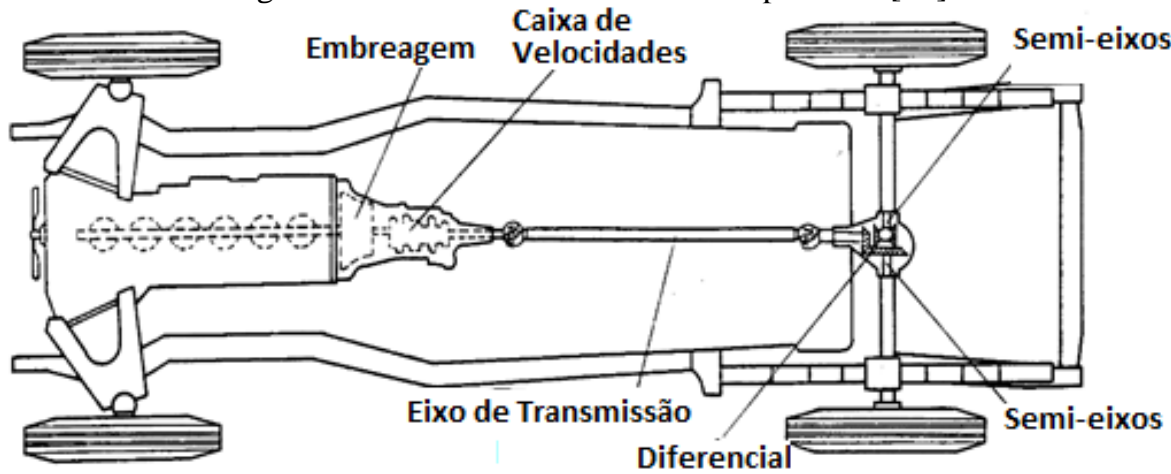

\section{MATERIAIS E MÉTODOS}

Para realizar o trabalho primeiramente foi feito um estudo do funcionamento e caraterísticas das caixas de velocidades mais utilizadas nos automóveis e posteriormente foi realizado um estudo comparativo das diferentes caraterísticas mais importantes de um mesmo tipo de modelo de carro os quais são fabricados com caixas manuais e automática.

O câmbio manual é a principal e mais simples forma de transmissão automotiva. Seu funcionamento está associado as relações de engrenagens da caixa de velocidades, que serão as responsáveis pela multiplicação do torque. Além disso, sua principal característica é a troca de marcha 


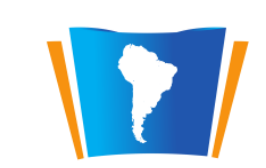

LATIN AMERICAN

manual em conjunto com o acionamento do pedal de embreagem. [17], como se pode observar na figura 3 .

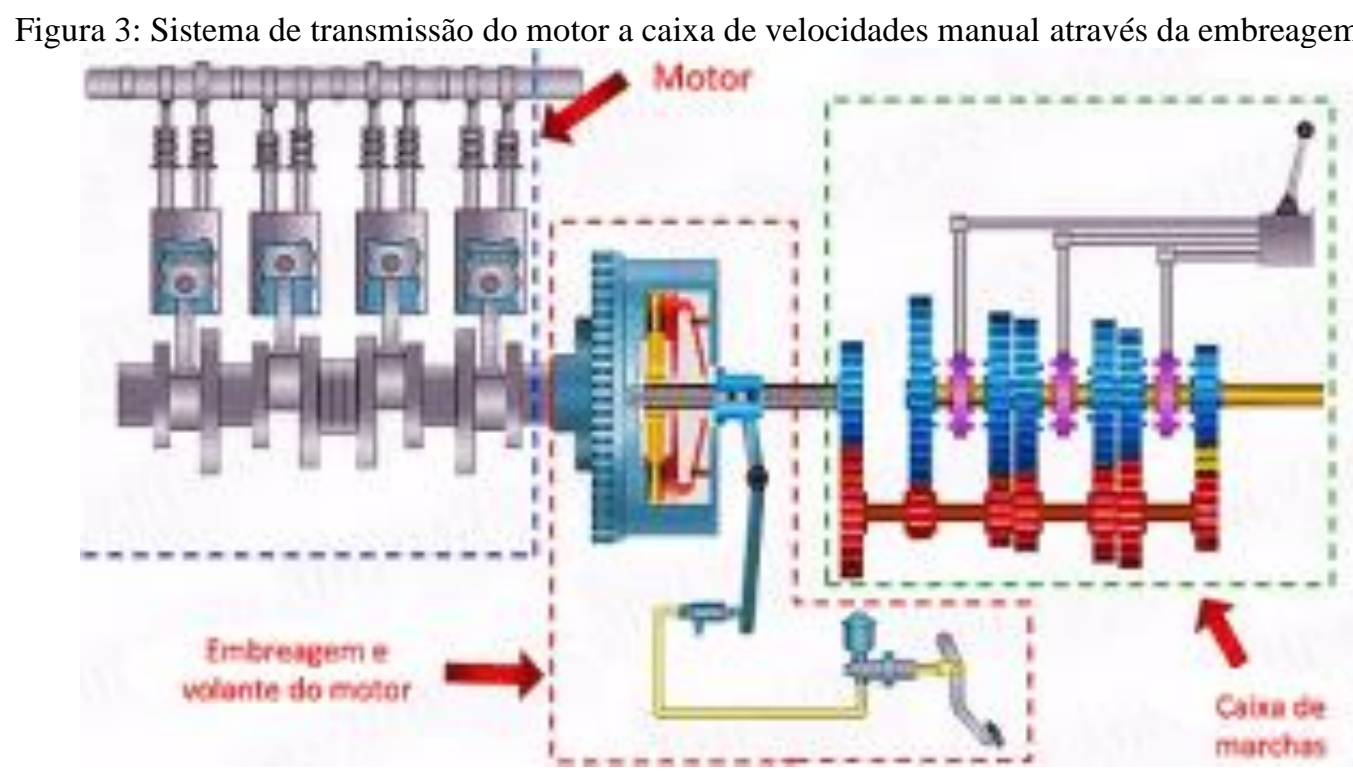

A caixa de velocidades manual é constituída, no seu interior, fundamentalmente, de forma simples, por dois veios e rodas dentadas formando alguns pares de engrenagens, conforme Figura 4. [18]. Para fazer a mudança de marcha acionamos o pedal da embreagem Figura 3) liberando o motor e fazemos mover o garfo (Figura 4) que vai fazer deslizar, horizontalmente, no veio amarelo, permitindo o engate das engrenagens pretendidas, criando-se assim a relação de transmissão definida pelo fabricante.

Figura 4: Caixa de velocidades manual [18]

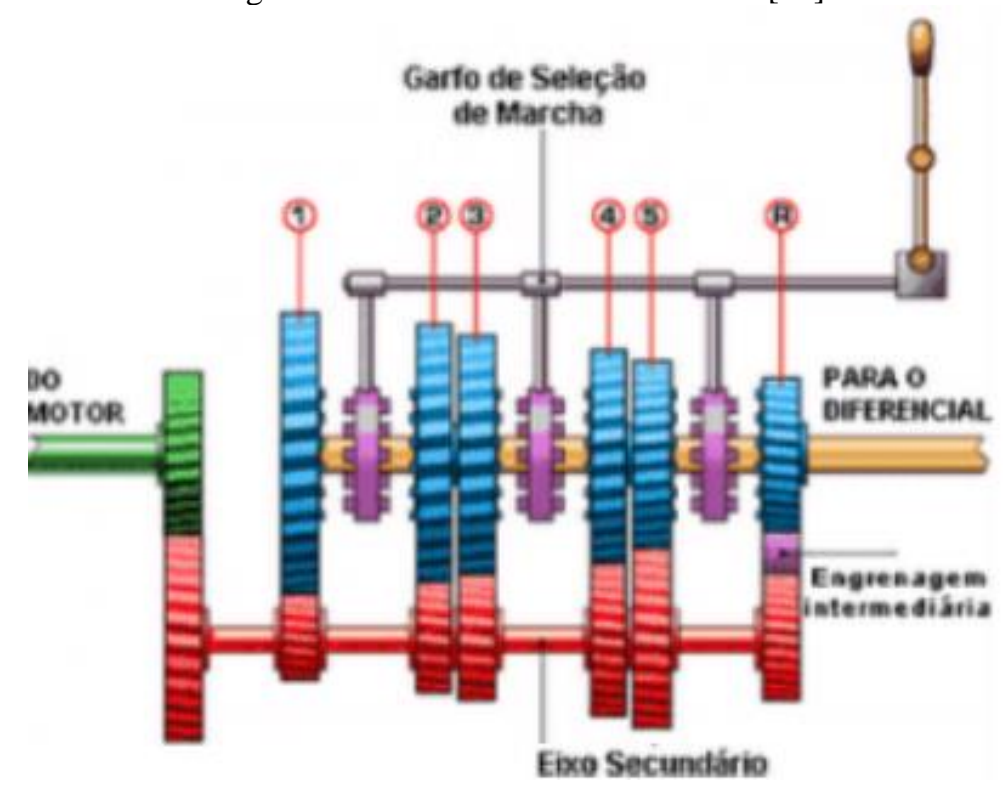




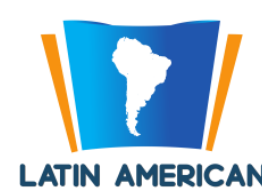
LATIN AMERICAN

O sistema de transmissão automática é um dos modelos que possuem crescimento mais notável nos últimos trinta anos. Seu funcionamento dispensa a utilização de pedais de embreagem e comando de alavanca seletora de marchas. Além disso, existem dois modelos popularizados em nosso contexto: câmbios automáticos de transmissão continuamente variável (CVT) e câmbios de transmissão por sistemas planetários. Estimativas feitas por consultoras especializadas afirmam que o câmbio automático é responsável por mais de cinquenta porcento das vendas de veículos em 2019. A chegada dos cambio automáticos em veículos denominados como "populares" contribuem para esse avanço em vendas, uma vez que os veículos com mecanismos automáticos eram reconhecidos pelo seu alto valor, embora oferecessem conforto a seus consumidores. [18-20]

A caixa automática não tem embreagem, como as manuais ou automatizadas, usa um conversor de binário como na figura 5a. A transmissão de potência não se faz mecanicamente, mas sim hidraulicamente, através de óleo especial, ATF (Automatic Transmission Fluid). O sistema de engrenagens, numa caixa automática, é, também, diferente de uma manual ou automatizada. Estas engrenagens são constituídas por 4 elementos: uma engrenagem central, a solar; as engrenagens planetárias, que giram em torno da engrenagem solar; a coroa (anular, na imagem) e o porta planetários (braço, na imagem) conforme ilustrado na Figura 5b.

Figura 5: Caixa de velocidades automática a)- conversor de binário, b) engrenagens [18].

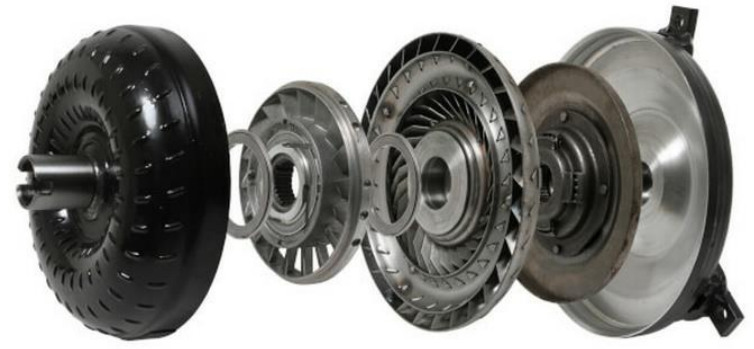

a)

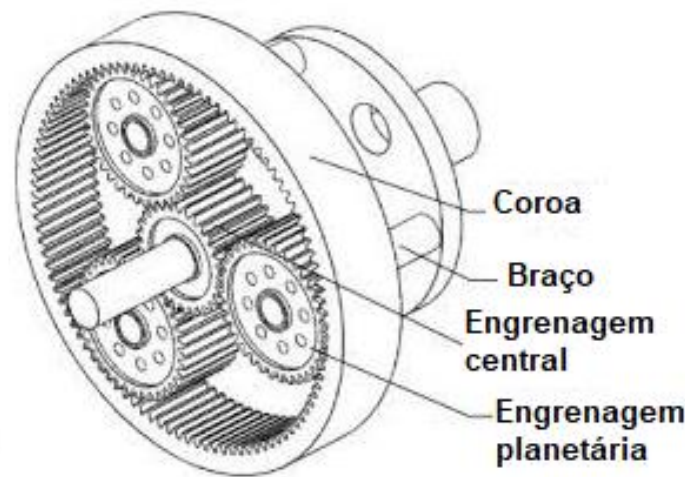

b)

A caixa de velocidades CVT (Continously Variable Transmission). Na sua génese temos duas polias cujos diâmetros de contacto com a corrente de transmissão variam em função da potência e do momento de força (torque) desejado. Estas duas polias estão ligadas entre si através de uma corrente metálica, conforme se pode observar na Figura 6. Uma polia recebe o movimento do motor e a outra transmite ao diferencial (veículos com tração traseira) ou ao veio de transmissão (veículos com tração dianteira). [18]

A relação de transmissão e, portanto, as velocidades, varia continuamente através da variação dos diâmetros de contacto das polias com a correia de transmissão. O momento de força a transmitir, 
do motor para as rodas motrizes, depende diretamente da pressão hidráulica aplicada às polias para fazer variar o seu diâmetro e da área de contacto da correia de transmissão com as polias, através da qual se gera o atrito necessário para evitar o escorregamento da correia de transmissão. Alguns fabricantes definem intervalos de velocidades virtuais, portanto, mostrados no painel de instrumentos, para que o condutor tenha noção de qual a mudança que estaria engrenada.

A grande vantagem destas caixas de velocidades reside na economia do consumo de combustível. Enquanto que na CVT temos uma constância das rpm nas trocas de mudanças, nas automáticas, ou manuais, existe uma variação significativa, como pode ser observado no gráfico da Figura 7 [18].
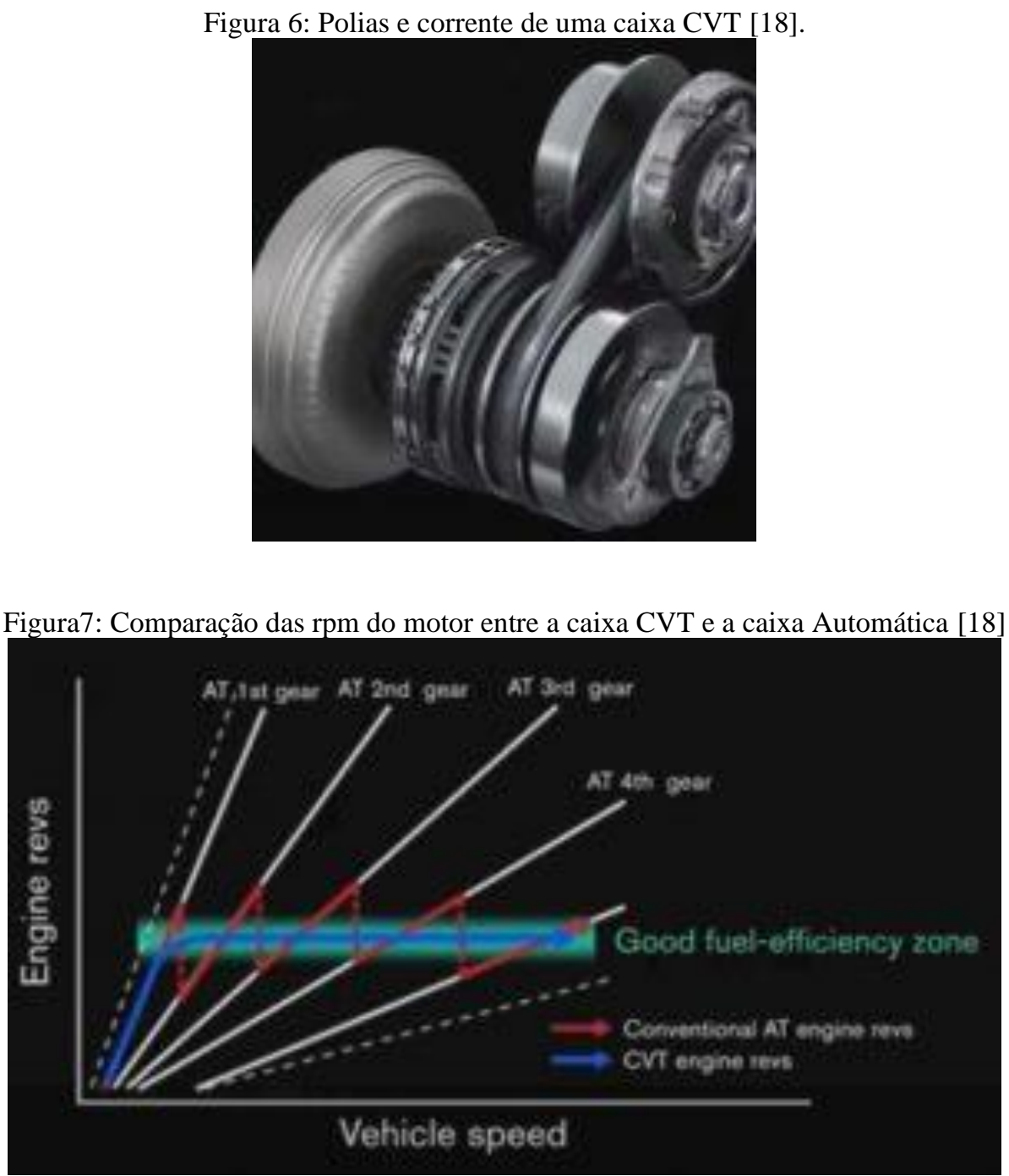

A caixa de velocidades automatizada, também conhecida por robotizada, pilotada, DSG (Direct Shift Gearbox), DCT (Dual Clutch Transmission), é uma caixa onde não há intervenção humana na 
gestão das mudanças. Em lugar de termos uma ligação mecânica entre a alavanca seletora de velocidades e a caixa para gerir a entrada e saída de mudanças esta gestão faz-se eletro-hidraulicamente por atuadores e, tal como nas manuais, por garfos (Figura 8). Estas caixas funcionam como automáticas uma vez que podem trocar as mudanças sem intervenção do condutor, embora o seu princípio de funcionamento seja diferente. [18]

O estudo foi realizado utilizando o modelo Chevrolet Onix 1.4 L, ano de 2018. A partir disso, foram retiradas informações fornecidas pelo fabricante, para as comparações. [21, 22]. Na tabela no. 1 podem se observar as especificações mais importantes desse modelo tanto para o carro manual como o automático.

Figura 8: Caixa de velocidades automatizada. [18]

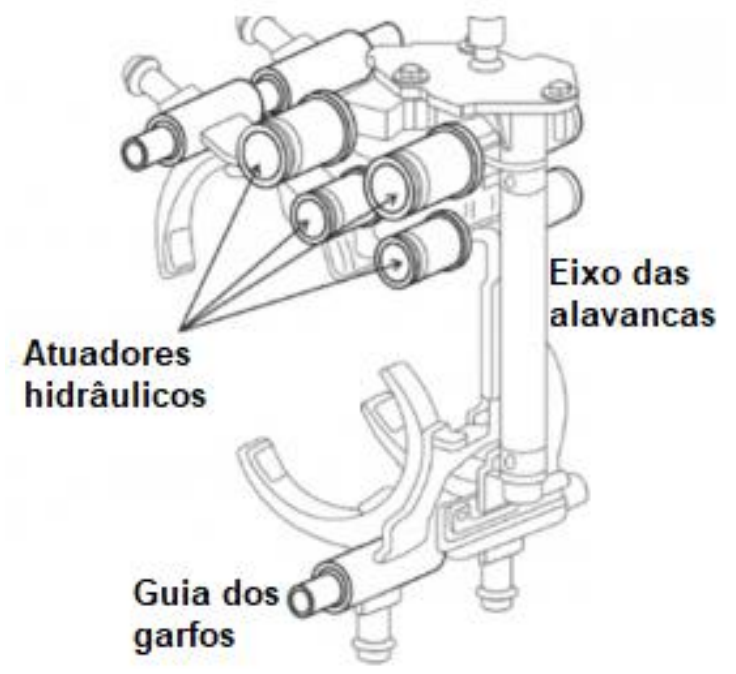

Tabela 1. Especificações Técnicas do Veículo [21].

\begin{tabular}{ccc}
\hline Transmissão & Automática & Manual \\
\hline Peso $(\mathrm{Kg})$ & 1467 & 1417 \\
Potência $(\mathrm{Cv})$ & 98 & 98 \\
rpm & 4800 & 4800 \\
Torque Máximo $(\mathrm{Kgfm})$ & 13,9 & 13,9 \\
Velocidade Máxima $(\mathrm{Km} / \mathrm{h})$ & 171 & 180 \\
\hline
\end{tabular}

A partir desses dados da tabela 1, as relações de transmissão da tabela 2 e a equação 1 , é possível determinar as velocidades e fazer o diagrama "Dente de Serra" utilizando como referência rotações por minuto levando em consideração o melhor torque a ser entregue pelo motor, que segundo informações do fabricante seria de 13,9 Kgfm que ocorre na rotação de referência de 4800 rpm.

$$
\text { Velocidade }=\frac{2 \cdot \pi \cdot N \cdot R_{\text {dinâmico }}}{60 \cdot i_{m} \cdot i_{\text {dif }}}
$$

Onde, $\boldsymbol{N}$, é a rotação do motor em rpm, $\boldsymbol{R}_{\text {dinâmico }}$ é o raio dinâmico do pneu. De acordo com 
os modelos de pneus fornecidos pelo manual [22], foi utilizado o raio dinâmico de 0,27 $\mathrm{m}$. Além disso, $\boldsymbol{i}_{\boldsymbol{m}}$ representa a relação de transmissão da marcha em questão (Tabela 2) [21] e $\boldsymbol{i}_{\boldsymbol{d i f}}$ representa a relação entre os conjuntos de engrenagens do diferencial, que é constante para cada caixa de marcha (Tabela 2) [21].

Tabela 1. Relação de Transmissão de Marchas [21]

\begin{tabular}{ccc}
\hline Marchas & Mecânico & Automático \\
\hline $1^{\mathrm{a}}$ & 3,73 & 4,44 \\
\hline $2^{\mathrm{a}}$ & 1,96 & 2,90 \\
\hline $3^{\mathrm{a}}$ & 1,32 & 1,89 \\
\hline $4^{\mathrm{a}}$ & 0,95 & 1,44 \\
\hline $5^{\mathrm{a}}$ & 0,76 & 1 \\
\hline $6^{\mathrm{a}}$ & 0,61 & 0,74 \\
\hline Ré & 3,63 & 4,63 \\
Diferencial & 4,63 & 4,11 \\
\hline
\end{tabular}

Para determinar a potência e posteriormente o desempenho para o Melhor Consumo é utilizada a equação 2

$$
P_{E}=\frac{P_{M E} \cdot A_{c i} \cdot d \cdot N}{1200}
$$

Em que $\boldsymbol{P}_{\boldsymbol{E}}$ representa a potência (em Kw/h), $\mathrm{P}_{\mathrm{ME}}$ é a pressão media, que se origina dentro da câmara de combustão no momento da explosão, $\left(\mathrm{A}_{\mathrm{ci}}\right.$. d) representa o volume deslocado pelo gás (em $\mathrm{dm}^{3}$ ), e $\boldsymbol{N}$ representam as rotações por minutos geradas no eixo do motor com o trabalho obtido.

Utilizando as equações anteriores e as informações de trocas por velocidade recomendadas pelo fabricante que são apresentadas na Tabela 3, obtém-se as rotações de maior eficiência para troca de marchas, tanto para o modelo Mecânico (Tabela 4) e Automático (Tabela 5), e Os diagramas de "Dente de Serra" obtidos podem ser observados nas figuras 9 e 10, os quais apresentam a relação de rotações por minutos e velocidade em $\mathrm{Km} / \mathrm{h}$, onde pode-se observar para cada ponto que relaciona o torque de referência e a velocidade máxima, há um declínio brusco vertical de rotação. Esse fenômeno está relacionado com troca de marcha do motor, que é conduzida pelo condutor do veículo. Ou seja, trocase a marcha, a velocidade mantém-se e reduz-se a rotação, uma vez que a queda de rotações é diretamente proporcionou às relações de transmissão. 
Tabela 3. Trocas de marcha para melhor consumo de combustível (Chevrolet Brasil, 2021)

\begin{tabular}{|c|c|c|c|c|c|}
\hline \multirow{4}{*}{$\begin{array}{c}\text { VELOCIDADE DE TROCA } \\
\text { DE MARCHA } \\
\text { RECOMENDADA (MT) }\end{array}$} & \multicolumn{5}{|c|}{ 1.4L SPE/4 ECO } \\
\hline & \multicolumn{4}{|c|}{ Condução na cidade } & \multirow{3}{*}{$\begin{array}{l}\text { Condução na } \\
\text { estrada }\end{array}$} \\
\hline & \multicolumn{2}{|c|}{ Motor frio } & \multicolumn{2}{|c|}{ Motor quente } & \\
\hline & $\begin{array}{l}\text { Leve ou } \\
\text { moderada (1) }\end{array}$ & $\begin{array}{l}\text { Aceleração } \\
\text { alta (2) (3) }\end{array}$ & $\begin{array}{l}\text { Leve ou } \\
\text { moderada (1) }\end{array}$ & $\begin{array}{l}\text { Aceleração } \\
\text { alta (2) (3) }\end{array}$ & \\
\hline $1 a>2 a$ & $15 \mathrm{~km} / \mathrm{h}$ & $15 \mathrm{~km} / \mathrm{h}$ & $14 \mathrm{~km} / \mathrm{h}$ & $14 \mathrm{~km} / \mathrm{h}$ & $14 \mathrm{~km} / \mathrm{h}$ \\
\hline $2 a>3 a$ & $33 \mathrm{~km} / \mathrm{h}$ & $33 \mathrm{~km} / \mathrm{h}$ & $24 \mathrm{~km} / \mathrm{h}$ & $24 \mathrm{~km} / \mathrm{h}$ & $24 \mathrm{~km} / \mathrm{h}$ \\
\hline $3 a>4 a$ & $40 \mathrm{~km} / \mathrm{h}$ & $53 \mathrm{~km} / \mathrm{h}$ & $37 \mathrm{~km} / \mathrm{h}$ & $53 \mathrm{~km} / \mathrm{h}$ & $37 \mathrm{~km} / \mathrm{h}$ \\
\hline $4 a>5 a$ & $55 \mathrm{~km} / \mathrm{h}$ & $70 \mathrm{~km} / \mathrm{h}$ & $49 \mathrm{~km} / \mathrm{h}$ & $70 \mathrm{~km} / \mathrm{h}$ & $49 \mathrm{~km} / \mathrm{h}$ \\
\hline $5 a>6 a$ & $66 \mathrm{~km} / \mathrm{h}$ & $76 \mathrm{~km} / \mathrm{h}$ & $56 \mathrm{~km} / \mathrm{h}$ & $76 \mathrm{~km} / \mathrm{h}$ & $66 \mathrm{~km} / \mathrm{h}$ \\
\hline
\end{tabular}

Tabela 4. Velocidade do modelo manual para melhor consumo

\begin{tabular}{ccccc}
\hline Marcha & $\mathbf{i}_{\mathbf{m}}$ & $\begin{array}{c}\text { Velocidade } \\
(\mathrm{km} / \mathrm{h})\end{array}$ & $\boldsymbol{N}$ & $\boldsymbol{i}_{\boldsymbol{d i f}}$ \\
\hline $1^{\mathrm{a}}$ & 3,7 & 0,0 & 0,0 & 4,6 \\
$1^{\mathrm{a}}$ & 3,7 & 15,0 & 2547,9 & 4,6 \\
$2^{\mathrm{a}}$ & 2,0 & 15,0 & 1338,0 & 4,6 \\
$2^{\mathrm{a}}$ & 2,0 & 24,0 & 2140,8 & 4,6 \\
$3^{\mathrm{a}}$ & 1,3 & 24,0 & 1441,8 & 4,6 \\
$3^{\mathrm{a}}$ & 1,3 & 37,0 & 2222,7 & 4,6 \\
$4^{\mathrm{a}}$ & 1,0 & 37,0 & 1599,7 & 4,6 \\
$4^{\mathrm{a}}$ & 1,0 & 49,0 & 2118,5 & 4,6 \\
$5^{\mathrm{a}}$ & 0,8 & 49,0 & 1694,8 & 4,6 \\
$5^{\mathrm{a}}$ & 0,8 & 56,0 & 1936,9 & 4,6 \\
$6^{\mathrm{a}}$ & 0,6 & 56,0 & 1554,6 & 4,6 \\
$6^{\mathrm{a}}$ & 0,6 & 79,0 & 2193,4 & 4,6 \\
\hline
\end{tabular}

Tabela 5. Velocidade do modelo automático para melhor consumo

\begin{tabular}{ccccc}
\hline Marcha & $\mathbf{i}_{\mathbf{m}}$ & $\begin{array}{c}\text { Velocidade } \\
(\mathrm{km} / \mathrm{h})\end{array}$ & $\boldsymbol{N}$ & $\boldsymbol{i}_{\text {dif }}$ \\
\hline $1^{\mathrm{a}}$ & 4,4 & 0,0 & 0,0 & 4,1 \\
$1^{\mathrm{a}}$ & 4,4 & 14,2 & 2547,9 & 4,1 \\
\hline $2^{\mathrm{a}}$ & 2,9 & 14,2 & 1664,1 & 4,1 \\
\hline $2^{\mathrm{a}}$ & 2,9 & 18,2 & 2140,8 & 4,1 \\
\hline $3^{\mathrm{a}}$ & 1,9 & 18,2 & 1391,8 & 4,1 \\
\hline $3^{\mathrm{a}}$ & 1,9 & 29,1 & 2222,7 & 4,1 \\
\hline $4^{\mathrm{a}}$ & 1,4 & 29,1 & 1699,9 & 4,1 \\
\hline $4^{\mathrm{a}}$ & 1,4 & 36,3 & 2118,5 & 4,1 \\
\hline $5^{\mathrm{a}}$ & 1,0 & 36,3 & 1466,5 & 4,1 \\
\hline $5^{\mathrm{a}}$ & 1,0 & 47,9 & 1936,9 & 4,1 \\
\hline $6^{\mathrm{a}}$ & 0,7 & 47,9 & 1432,0 & 4,1 \\
\hline $6^{\mathrm{a}}$ & 0,7 & 73,4 & 2193,4 & 4,1 \\
\hline
\end{tabular}


Conforme Cardoso (2012) [23], um dos parâmetros mais utilizados nos últimos anos para se medir e reduzir o consumo de combustível é o dimensionamento do parâmetro. "Ratio Spread", o qual é a relação que há entre duas marchas adjacentes calculada pela razão entre as relações de transmissão. Esse valor pode ser obtido pela Equação (3).

$$
\text { Ratio }_{\text {spread }}=\frac{i_{a}}{i_{b}}
$$

Onde $i_{a}$ é a relação de transmissão com razão superior, representante da marcha "a", e $i_{b}$ a relação com razão inferior, representando a marcha "b”. O “Overall Ratio Spread” é uma variável pertencente a mesma família, todavia representa um termo global, e é calculado utilizando também a equação (3). Esse parâmetro consiste na razão entre a relação de transmissão da primeira marcha pela última, como $1^{\mathrm{a}}$ e $6^{\mathrm{a}}$ correspondente para o Chevrolet Onix 2018, 1.4 L, como a primeira relação de transmissão é 4,4: 1 e a última é 0,7:1, o Overall Ratio Spread seria, conforme a equação (3): relação de $1^{\text {a }}(4,4) /$ relação de $6^{\mathrm{a}}(0,7)=6,29$. Para um melhor consumo e desempenho, faz-se necessário um “Overall Ratio Spread” elevado, pois teríamos uma alta aceleração inicial e altas velocidades nas últimas marchas. É possível associar esse fenômeno com a características de câmbios de marchas longas e curtas. Por esse motivo, a maioria dos veículos com câmbios automáticos e manuais adotam o chamado escalonamento misto. Ou seja, possuem marchas curtas para a retirada da inércia e marchas longas para o alcance de significantes taxas de velocidades a rotações não muito elevadas [24]. Para este estudo utilizando o Chevrolet Onix, foi realizado um diagrama de Ratio Spread para conferir o consumo, utilizando a Equação (3) e os dados de relações de transmissão da tabela 2, cujos resultados encontram-se relacionados na Tabela 6 e no gráfico da Figura 11.

Tabela 2. Relação de Ratios spreads para o modelo

\begin{tabular}{ccc}
\hline Troca & Manual & Automâtico \\
\hline $1->2$ & 1,9 & 1,5 \\
$2->3$ & 1,5 & 1,5 \\
$3->4$ & 1,4 & 1,3 \\
$4->5$ & 1,3 & 1,4 \\
$5->6$ & 1,2 & 1,4 \\
\hline Overall & 6,2 & 6,3 \\
\hline
\end{tabular}

\section{RESULTADOS E DISCUSSÃO}

Para cada ponto que relaciona o torque de referência e a velocidade máxima, há um declínio brusco vertical de rotação. Esse fenômeno está relacionado com troca de marcha do motor. Ou seja, troca-se a marcha, a velocidade mantém-se e reduz-se a rotação, uma vez que a queda de rotações é 
diretamente proporcionou às relações de transmissão. Ao se analisar o diagrama, é possível observar primeiramente que para cada troca de marcha efetuada, o veículo que porta câmbio manual atinge velocidades maiores em todos as relações de transmissão. Além disso, a velocidade máxima, conforme o diagrama dente de serra, calculada a rotações em torno de 4800 giros estaria em torno de $173 \mathrm{~km} / \mathrm{h}$ no veículo com câmbio manual e $161 \mathrm{~km} / \mathrm{h}$ no veículo automático.

Figura 9. Gráfico de desempenho para o melhor torque

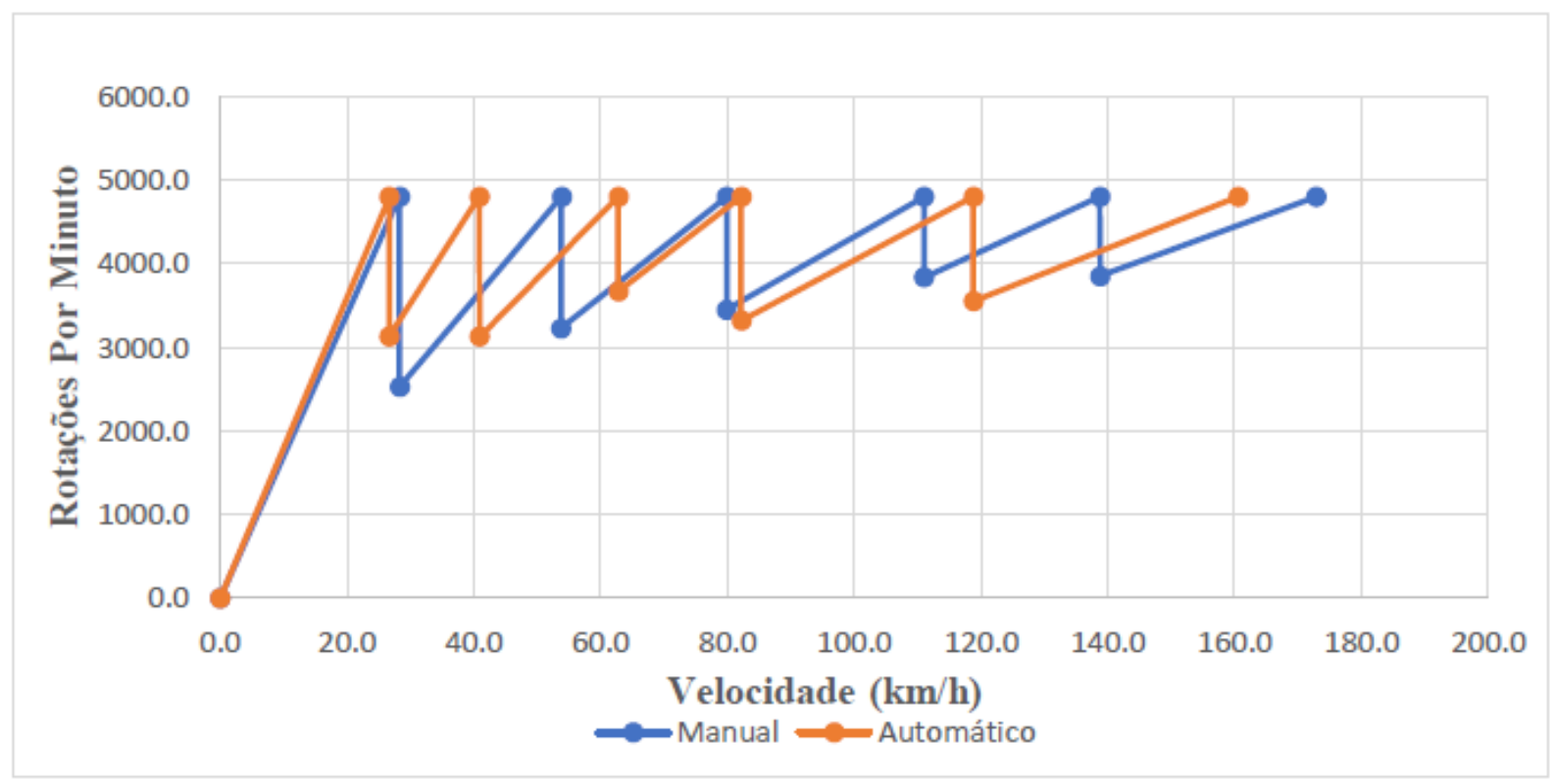

A tabela 6 mostra as velocidades na perspectiva da análise de desempenho. Pode-se observar como as velocidades, principalmente nas marchas 3, 4, 5 são superiores. Ou seja, para todas as relações de transmissões observa-se que o modelo equipado com versão manual possui melhor desempenho. Além disso, a velocidade máxima, conforme o diagrama dente de serra, calculada a rotações em torno de 4800 giros estaria em torno de $173 \mathrm{~km} / \mathrm{h}$ no veículo com câmbio manual e $161 \mathrm{~km} / \mathrm{h}$ no veículo automático.

Tabela 6. Velocidades do modelo para o melhor torque

\begin{tabular}{cccc}
\hline Marcha & Automático $(\mathbf{K m} / \mathbf{h})$ & Manual $(\mathbf{K m} / \mathbf{h})$ & Diferença \\
\hline $1^{\mathrm{a}}$ & 0 & 0 & $0 \%$ \\
$2^{\mathrm{a}}$ & 26,7 & 28,3 & $6 \%$ \\
$3^{\mathrm{a}}$ & 41 & 53,8 & $31 \%$ \\
$4^{\mathrm{a}}$ & 62,8 & 80 & $27 \%$ \\
$5^{\mathrm{a}}$ & 82,2 & 111 & $35 \%$ \\
$6^{\mathrm{a}}$ & 118,8 & 138,8 & $17 \%$ \\
\hline
\end{tabular}


A Tabela 7 mostra as rotações por minuto calculadas para as condições do modelo para o melhor torque, tanto para o carro manual como automático em cada troca de marcha e na figura 10 é mostrado o gráfico de Dente de Serra para o melhor consumo de combustivel. Pode-se observar na Tabela 7 que no veículo manual há uma tendência de aumento progressivo nas rotações a cada troca e no veículo automático por outro lado, apresenta uma variação de rotação em faixas semelhantes. Podem-se observar variações semelhantes, no anali-se em função do consumo otomo em relação à comparação com o torque ótimo, em todos os parâmetros: nas velocidades, nas rotações e e nos comprimentos das marchas, entretanto com taxas de variação menores.

A Tabela 7. Rotações por minuto do modelo para o melhor torque

\begin{tabular}{cccc}
\hline Marcha & Manual & Automático & Diferença \\
\hline $1^{\mathrm{a}}$ & 0 & 0 & 0 \\
$2^{\mathrm{a}}$ & 2524,3 & 3136,7 & $24,4 \%$ \\
$3^{\mathrm{a}}$ & 3231,9 & 3127,8 & $3,3 \%$ \\
$4^{\mathrm{a}}$ & 3454,4 & 3668,6 & $5,2 \%$ \\
$5^{\mathrm{a}}$ & 3839,2 & 3320,8 & $15,6 \%$ \\
$6^{\mathrm{a}}$ & 3853,2 & 3551,5 & $9,4 \%$ \\
\hline
\end{tabular}

Figura 10. Gráfico de desempenho para o melhor consumo

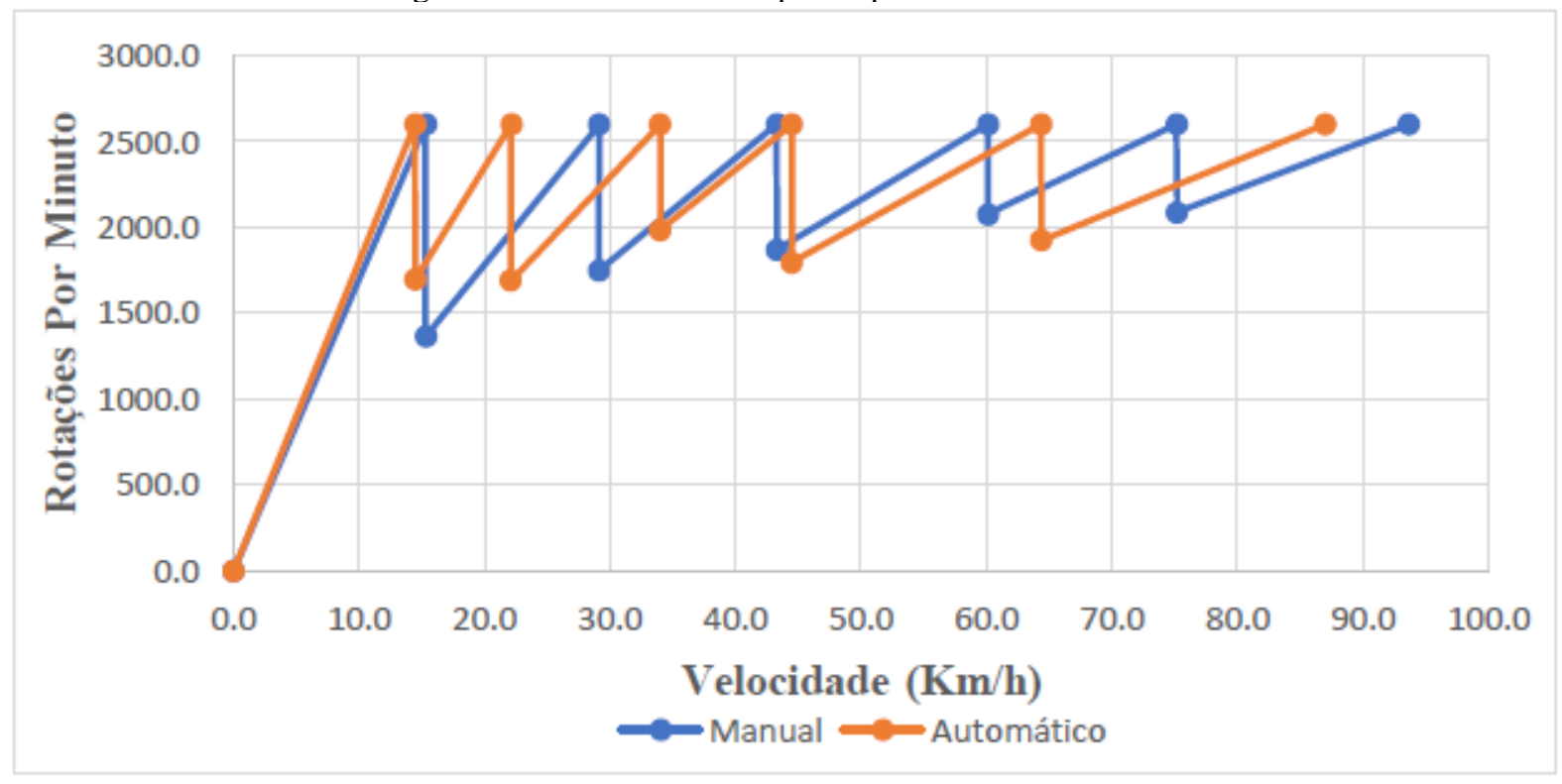

Os principais parâmetros envolvendo diferença de desempenho na velocidade e rotação são explicados pela tendência de marchas longas no modelo equipado com câmbio manual e, no caso do veículo automático, pela tendência de marchas mais curtas. Segundo Guerra (2015) [24], essa distinção de comprimento de marchas, é dada conforme a dimensão das rodas dentadas que compõe a transmissão. Ou seja, a divisão das medidas da Coroa e do Pinhão, também conhecido como relação 
de transmissão (ilustrada na tabela 2), uma vez que estamos tratando de transmissões por engrenagens. Nesse sentido, quando se têm relações de marchas muito próximas, fica caracterizado um veículo com comprimento menor, ou seja, as diferenças de razão entre suas engrenagens (diferença das relações de transmissão para todas as marchas) não são tão acentuadas. Por conseguinte, quando se têm relações mais longas, é possível observar maior defasagem. Geralmente, esse fenômeno é responsável pela influência no comportamento do desempenho do carro: tempo de trocas de marchas, taxas de velocidade, índices de aceleração, quedas de rotação nas trocas etc.

Figura 11: Relação de Ratios spreads para os modelos Manual e Automâticos

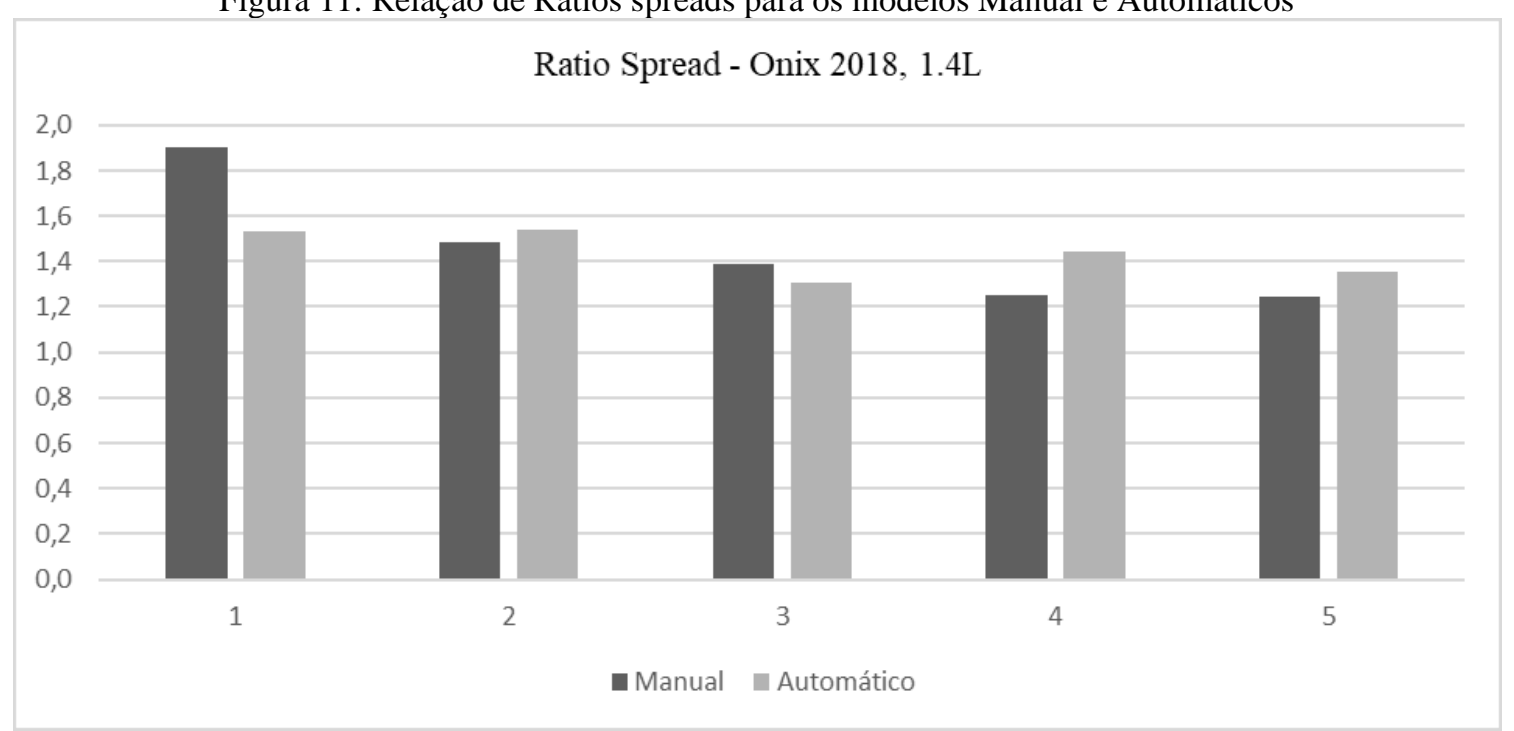

\section{CONCLUSÕES}

Neste estudo foi realizada uma análise técnica, utilizando o modelo Chevrolet Onix 2018, 1.4, automático em comparação com o modelo manual utilizando princípios e equações mecânicas e dados técnicos do fabricante e informações na bibliografia de trabalhos anteriores

Em relação ao desempenho o veículo manual apesentou melhores caraterísticas em todos os sentidos relacionados ao câmbio automático, como: maiores velocidades finais e maior tempo de trocas entre as marchas. No caso específico do veículo automático, foi possível visualizar um veículo mais atento às acelerações, o que acaba por gerar troca de marchas mais curtas, devido à alta multiplicação de torque inicial, que é necessária por ser de um peso maior do que o modelo manual (5\% a mais)

Em relação ao consumo de combustível foi constatada maior economia no modelo manual que no automático embora se tenham analisado valores bem próximos. O modelo automático apresenta maiores perdas em sua eficiência de consumo, principalmente, devido ao conversor de torque e por suas trocas estarem relacionadas com rotações pré-estabelecidas. 
Os veículos automáticos apresentaram um custo de manutenção superiores a um $10 \%$ do que o modelo manual e um valor de compra de $9 \%$ a mais do valor de compra do modelo manual, mas em relação às desvalorizações por dois anos de uso, percebeu-se dados bem próximos na Tabela, sendo que, o veículo automático superava a valorização em $2 \%$ em relação ao modelo manual.

Em relação ao modelo equipado com o câmbio automático, é possível verificar: maior conforto e possibilidade de acessibilidade a condutores com condições especiais, com uma tecnologia superior; e melhor ergonomia. 


\section{REFERÊNCIAS}

[01]- Shibata, R. M.. Engenharia de produtos: desenvolvimento de veículos globais em subsidiária brasileira de multinacional / Dissertação de Mestrado- Centro Universitário FEI, São Bernardo do Campo, 2010.

[02]- Lechner, G.; Naunheimer, H. Automotive transmission: fundamentals, selection, design and application. Sttutgart: Springer, 1999.

[03]- Padilha, J. L. Sistemas de Transmissão Automotiva. 1. ed. Brasília: NT, v. 1, 2018.

[04]- Saeed A. B. Simulation design of an Intelligent system for Automotive_transmission Gearbox Based on FPGA. International Journal of Engineering and Technology Vol.6(2), 2018.

[05]- Silva A. R. V., Macêdo N.J. C., Miranda A. G., Verçosa L. A., Garcia del Pino G., Rodrigues R. A., Nascimento D. A., Influência do tratamento térmico de normalização na microestrutura e propriedades mecânicas do aço SAE 1035 utilizado em motocicletas, ATENAS, Doi: 10.22533/at.ed.6222105043, 2021.

[06]- Mendes C. S., Garcia del Pino G., Bezazi A., Boumediri H., Neto J. C., Torres A. R., Kieling A. C., Garcia S. D. Comparative performance comparison of Toolox44 tool steel versus SAE H13 for hot forging. Latin American Journal of Development. v. 3, n. 3, p. 1181-1197. Doi: 10.46814/lajdv3n3014. 2021.

[07]- Filizzola D. M., Santos T. S., Miranda A. G., Costa J. C. M., Nascimento N. R, Santos M. D., Bello R.H., Garcia del Pino G., Macedo N. J. C. Annealing Effect on the Microstructure and Mechanical Properties of AA 5182 Aluminum Alloy. Materials Research.; 24(4); 2021; DOI: 10.1590/1980-5373-MR-2020-05

[08]- SENAI, Serviço Nacional De Aprendizagem Industrial. Mecânica veículos leves: Sistema automática. São Paulo: SENAITEC. 2002.

[09]- Goiés, M. Carro com câmbio manual pode virar raridade no Brasil; entenda as razões.

Disponível em: https://www.uol.com.br/carros/noticias/redacao/2020/01/16/carr. Acesso em: 14 de julho 2021.

[11]- JATCO .Corporate History, $2020 . \quad$ Disponivel em: https://www.jatco.co.jp/english/company/history.html>. Acesso em: 18 Maio 2021

[12]- Moreira I. S.; Ruwer L. M. E.; Mundo do trabalho, serviço social e desenvolvimento sustentável; Latin American Journal of Development, v. 2, n. 5; p. 206-227; 2020.

[13]- Garcia del Pino G., Bezazi A., Boumediri H., Kieling A. C., Silva C. C., Dehaini J., Rivera J. L. V., Valenzuela M. G. S., Diaz F. R. V., Panzera T, H., Hybrid epoxy composites made from treated curauá fibres and organophilic clay. Journal of Composite Materials, 1(0) 1-13, (2020), DOI: $10.1177 / 0021998320945785$.

[14]- Garcia del Pino G., Kieling A. C., Bezazi A., Panzera T. et al. Hybrid polyester composites reinforced with curauá fibres and nanoclays, Fibres and Polymers, 21, No.2, 399-406, (2020). 
[15]- Melconian, S. Elementos de Máquina. Érica 10. ed. [S.1.], 2018.

[16]- Halliday; R. Fundamentos de Física. Mecânica. 9. ed. [S.1.]: LTC, v. 1, 2012.

[17]- EEEP. Curso Técnico em Manuntenção Automotiva. [S.1.]: Escola Estadual de Educação Profissional, 2014.

[18]- https://pinkfox.pt/category/caixas-de-velocidades/. Acesso em: 01/07/2021

[19]- Erjavec, J. Automatic Transmissions. 1. ed. [S.1.]: Cengage Learning, v. I, 2004.

[20]- Felix, L. Carro automático salta de $12 \%$ para 50\% do mercado brasileiro em 10 anos. Quatro Rodas, 2020. Disponivel em: https://quatrorodas.abril.com.br/noticias/carroautomatico-salta-de-12para-50-do-mercado-brasileiro-em-10-anos/. Acesso em: 01/07/2021

[21]- Chevrolet Brasil. Manuais do Proprietário. Meu.Chrvrolet, 2021. Disponivel em: https://meu.chevrolet.com.br/saiba-mais-e-suporte/manuais-guias>. Acesso em: 17 de Agosto de 2021.

[22]- Chevrolet Brasil. Peça Chevrolet. 2021. Disponível em: https://www.pecachevrolet.com.br. Acesso em: 17 de Agosto de 2021.

[23]- Cardoso, Júlio Cezar Sartoreli. Estudo para verificar a influência das Transmissões Automáticas modernas de veículos de passeio no consumo de combustível. Monografia de Especialização- Centro Universitário do Instituto Mauá de Tecnologia, São Caetano do Sul, São Paulo, 2012.

[24]- Guerra, P. Câmbio curto e câmbio longo: entenda a influência no desempenho do veículo automobilistico. Educação Automotiva, 2015. Disponível em:

https://educacaoautomotiva.com/2015/08/05/cambio-curto-e-cambio-longo-entenda-ainfluencia-nodesempenho-do-carro. Acesso em: 25 maio 2021 